\title{
Research on Renal Injury in Hashimoto's Thyroiditis Patients with Different Thyroid Function
}

\author{
Rongqin Dai ${ }^{1}$, Qian $\mathrm{Xu}^{1}$, Yang Chen ${ }^{1}$, Ying $\mathrm{Li}^{1}$, Chao Shen ${ }^{1}$, Jinbiao Zhang ${ }^{2, *}$ \\ ${ }^{1}$ Department of Medical Technology Laboratory, Cangzhou Medical College, Cangzhou, China \\ ${ }^{2}$ Laboratory Diagnostics Division, Cangzhou Hospital of Integrated Traditional Chinese and Western Medicine, Cangzhou, China
}

Email address:

zjb7829325@163.com (Jinbiao Zhang)

${ }^{*}$ Corresponding author

\section{To cite this article:}

Rongqin Dai, Qian Xu, Yang Chen, Ying Li, Chao Shen, Jinbiao Zhang. Research on Renal Injury in Hashimoto's Thyroiditis Patients with Different Thyroid Function. Science Journal of Public Health. Vol. 9, No. 3, 2021, pp. 98-102. doi: 10.11648/j.sjph.20210903.16

Received: April 13, 2021; Accepted: May 19, 2021; Published: May 24, 2021

\begin{abstract}
Objective: To assess the relationship between thyroid function and renal injury in Patients with Hashimoto's Thyroiditis. Method: To select 156 diagnosed HT patients as the research object, according to whether there is renal injury, they were divided into renal injury group with 21 cases and non-renal injury group with 135 cases. According to the thyroid function, they were divided into HT hyperthyroidism group (Group A) with 19 cases, normal HT thyroid function group (Group B) with 37 cases, HT subclinical hypothyroidism group (Group C) with 61 cases, and HT hypothyroidism group (Group D) with 39 cases. Besides, 40 cases of healthy check-up people are selected as the control group. ECLIA is adopted to test the thyroid function index of each group, Serum cystatin $\mathrm{C}$ and 24-hour urinary microalbumin were measured by immunoturbidimetry, serum creatinine was measured by creatine oxidase method, and the endogenous creatinine clearance rate was calculated. Result: TSH, TPOAb and TGAb in renal injury group were distinctly higher than those in non-renal injury group, while FT3 and FT4 were distinctly lower than those in non-renal injury group; Comparison of serum creatinine in Hashimoto groups, Group D is significantly higher than other groups, Group C is obviously higher than Group A, Group B, and the control group, Group B is obviously higher than Group A, Group A is significantly lower than other groups; Comparison of cystatin C and creatinine clearance of each group, Group D is significantly lower than other groups, Group C is obviously lower than Group A, Group B, and the control group, Group B is obviously lower than Group A, Group A is significantly higher than other groups; Comparison of 24-hour urinary microalbumin of each group, Group D is significantly higher than other groups, Group C is obviously higher than Group A, Group B, and the control group, and the differences have statistic significance $(\mathrm{P}<0.05)$. The correlation analysis shows that FT3, FT4 are in negative correlation with serum creatinine and in positive correlation with cystatin $\mathrm{C}$ and creatinine clearance; TSH, TPOAb, TGAb is in positive correlation with 24-hour urinary microalbumin. Conclusion: There are obvious differences in renal function indexes of HT patients with different thyroid function states, which may be related to the effect of thyroid hormones and autoantibodies.
\end{abstract}

Keywords: Hashimoto's Thyroiditis, Thyroid Function, Renal Injury

\section{Introduction}

Autoimmune thyroid diseases (AITD) mainly include Hashimoto's thyroiditis (HT), Graves disease, autoimmune atrophic thyroiditis, postpartum thyroiditis, painless thyroiditis, thyroid associated ophthalmopathy, etc [1]. Graves disease and HT are the most common in the clinic [2]. As confirmed in previous studies, thyroid and renal are closely related, coordinate and influence each other [3]. There are frequent reports that renal injury caused by thyroid disease is misdiagnosed as diabetic nephropathy, primary nephrotic syndrome, lupus nephritis and interstitial nephritis. If kidney damage caused by thyroid disease can be early detected and the primary disease can be actively treated, the prognosis of kidney damage is good, and renal function can be partially or completely restored. However, if the diagnosis and treatment of the disease is delayed, irreversible changes will occur in the kidney [4]. AITD is an organ specific autoimmune disease. The immune function of patients is abnormal. The autoimmune 
system attacks the kidney and secondary kidney disease. Kidney damage even occurs before the symptoms of thyroid disease. A variety of antibodies against thyroid tissue can be detected in the blood of patients with AITD, and thyroid antibodies can also be detected in renal biopsy of patients with AITD related nephropathy. At present, there are many reports of renal injury associated with Graves disease, but few reports of renal injury associated with Hashimoto's thyroiditis. HT patients often present different thyroid dysfunction. Early patients present normal thyroid function or transient hyperthyroidism. With the progress of the disease, their thyroid function gradually manifests as hypothyroidism [5]. A large number of studies have shown that hypothyroidism can directly or indirectly affect the glomerular filtration rate, and other studies have reported that the prevalence of renal disease in patients with hyperthyroidism will also increase. By analyzing the changes of renal function indexes in HT patients with different thyroid function, this paper discusses the relationship between Hashimoto's thyroiditis and renal injury, aiming to provide a theoretical basis for the early diagnosis and treatment of HT complicated with renal disease.

\section{Methods}

\subsection{General Information}

156 patients who were clinically diagnosed with HT in the endocrinology department of Cangzhou Hospital of Integrated Traditional Chinese and Western Medicine, Hebei province from March, 2018 to December, 2019 were chosen to be study objects, among which there were 31 men cases, 125 women cases with age from 22 69, and the average age were (45.61 \pm 17.28$)$ years old.

According to whether there is renal injury, they were divided into renal injury group with 21 cases and non-renal injury group with 135 cases. According to the thyroid function, they were divided into HT hyperthyroidism group (Group A) with 19 cases, normal HT thyroid function group (Group B) with 37 cases, HT subclinical hypothyroidism group (Group C) with 61 cases, and HT hypothyroidism group (Group D) with 39 cases. Besides, 40 cases of healthy check-up people are selected as the control group, in which there were 8 men cases and 32 women cases with age from $22 \sim 67$, and the average age were $(45.33 \pm 16.48)$ years old. This research has been approved by the ethics committee and all the subjects were informed and agreed.

\subsection{Diagnostic Criteria}

\subsubsection{Diagnostic Criteria of Hashimoto's Thyroiditis}

The HT diagnosis refers to the diagnostic criteria in the 8th version of Internal Medicine published by the People's medical publishing house [6], including: (1) The one with clinical features, such as diffusing goiter, tough texture, especially with thickening of isthmus and pyramidal lobe, etc., and Thyroid peroxidase antibody (TPOAb) or Thyroglobulin antibody (TGAb) positive; (2) The one without goiter, but with distinct TPOAb or TGAb increase as well as hypothyroidism.

\subsubsection{Diagnostic Criteria of Thyroid Dysfunction}

The diagnostic criteria of thyroid dysfunction including [7]: (1) Hyperthyroidism: TSH $<0.27 \mathrm{mU} / \mathrm{L}$, FT3 $>7.1 \mathrm{pmol} / \mathrm{L}$, FT4 $>22$ pmol/L; (2) Subclinical hypothyroidism: TSH $>4.2$ mU/L, FT3 and FT4 are normal; (3) Hypothyroidism: TSH > $4.2 \mathrm{mU} / \mathrm{L}, \mathrm{FT} 3<2.8 \mathrm{pmol} / \mathrm{L}$ and $/$ or FT4 $<12 \mathrm{pmol} / \mathrm{L}$.

\subsubsection{Diagnostic Criteria of Renal Injury}

The criteria of renal injury: creatinine clearance (Ccr) less than $80 \mathrm{ml} / \mathrm{min}$ or 24-hour urinary microalbumin more than $30 \mathrm{mg}$.

\subsection{Experimental Method}

Morning fasting venous blood is taken from all the subjects and put into pro-coagulation tube. Adopt Cobas E601 electrogenerated-chemiluminescence analyzer of Roche supporting assay to test thyroid function and antibody indicator, including TSH, FT3, FT4, TPOAb and TGAb; Adopt Hitachi 7600 automatic biochemical analyzer to test serum cystatin $\mathrm{C}$ (Cys-C) by immunoturbidimetry, Adopt Hitachi 7600 automatic biochemical analyzer to test serum creatinine by creatine oxidase method, C-G formula for calculating Ccr: $\mathrm{Ccr}($ male $)=(140-$ age $) *$ body weight $(\mathrm{kg}) /$ $(0.818 *$ serum creatinine). If female, the above results were multiplied by 0.85 .

The urine in the bladder of the testee was emptied and discarded at 8 a.m., and then the urine discharged each time was collected till 8 a.m. the next day, which was 24 -hour urine. All the urine was put into a container, and the urine volume was recorded. After mixing the urine, $3 \mathrm{ml}$ of urine was extracted. The concentration of urinary albumin was determined by immunoturbidimetry with Hitachi 7600 automatic biochemical analyzer. The result multiplied by urine volume is 24 hours urine micro albumin volume.

\subsection{Statistic Method}

The data adopts SPSS24.0 software for analysis and processing, the metering data is expressed by $\left(x_{ \pm s}\right)$, the comparison among multiple groups adopts analysis of variance, pairwise comparison adopts SNK-q test, and correlation analysis adopts linear correlation analysis.

\section{Results}

\subsection{The Correlation Between Thyroid Function Index and Renal injury in Hashimoto's Thyroiditis Patients}

$\mathrm{TSH}, \mathrm{TPOAb}$ and TGAb in renal injury group were distinctly higher than those in non-renal injury group, while FT3 and FT4 in renal injury group were distinctly lower than those in non-renal injury group. Those differences were statistically significant $(\mathrm{P}<0.05)$. (Table 1 ) 
Table 1. The correlation between thyroid function index and renal injury in Hashimoto's thyroiditis patients.

\begin{tabular}{|c|c|c|c|c|c|c|}
\hline Groups & $\mathbf{n}$ & TSHmIU/L & FT3pmol/L & FT4pmol/L & TPOAbIU/ml & TGAbIU/ml \\
\hline renal injury group & 21 & $19.12 \pm 9.36$ & $2.13 \pm 0.92$ & $9.45 \pm 3.14$ & $372.23 \pm 168.36$ & $782.74 \pm 154.86$ \\
\hline non-renal injury group & 135 & $3.23 \pm 1.56$ & $5.83 \pm 1.64$ & $16.23 \pm 2.76$ & $285.29 \pm 132.36$ & $653.52 \pm 129.47$ \\
\hline$t$ & & 4.72 & 3.68 & 4.03 & 3.85 & 3.47 \\
\hline$p$ & & 0.017 & 0.039 & 0.025 & 0.031 & 0.045 \\
\hline
\end{tabular}

\subsection{Comparison of Renal Function Indexes in Hashimoto Groups}

Comparison of serum creatinine in Hashimoto groups, Group D is significantly higher than other groups, Group $\mathrm{C}$ is obviously higher than Group A, Group B, and the control group, Group B is obviously higher than Group A, Group A is significantly lower than other groups, and the differences have statistic significance $(\mathrm{P}<0.05)$, as for the comparison of Group $\mathrm{B}$ with the control group, the difference has no statistic significance $(\mathrm{P}>0.05)$; Comparison of $\mathrm{Cys}-\mathrm{C}$ and creatinine clearance of each group, Group D is significantly lower than other groups, Group C is obviously lower than Group A,
Group B, and the control group, Group B is obviously lower than Group A, Group A is significantly higher than other groups, and the differences have statistic significance $(\mathrm{P}<0.05)$, as for the comparison of Group B with the control group, the difference has no statistic significance $(\mathrm{P}>0.05)$; Comparison of 24-hour urinary microalbumin of each group, Group D is significantly higher than other groups, Group C is obviously higher than Group A, Group B, and the control group, and the differences have statistic significance $(\mathrm{P}<0.05)$, as for the comparison of Group A and Group B with the control group, the difference has no statistic significance $(\mathrm{P}>0.05)$. (Table 2)

Table 2. Comparison of Each Group of Renal Function Index $(\bar{x} \pm s)$.

\begin{tabular}{llllll}
\hline Groups & n & Cys-C $(\mathbf{m g} / \mathbf{L})$ & Serum creatinine $(\boldsymbol{\mu m o l} / \mathbf{L})$ & Ccr $(\mathbf{m l} / \mathbf{m i n})$ & $\mathbf{2 4 - h o u r ~ u r i n a r y ~ m i c r o a l b u m i n ~}(\mathbf{m g})$ \\
\hline group A & 19 & $0.77 \pm 0.33$ & $56.82 \pm 14.87$ & $127.78 \pm 27.79$ & $9.06 \pm 2.65$ \\
group B & 37 & $0.62 \pm 0.21$ & $62.32 \pm 14.42$ & $109.56 \pm 15.12$ & $8.76 \pm 2.37$ \\
group C & 61 & $0.57 \pm 0.26$ & $71.27 \pm 18.94$ & $97.63 \pm 20.46$ & $19.86 \pm 3.59$ \\
group D & 39 & $0.51 \pm 0.28$ & $83.91 \pm 20.16$ & $81.45 \pm 22.16$ & $32.78 \pm 5.37$ \\
normal control group & 40 & $0.63 \pm 0.17$ & $64.57 \pm 14.23$ & $108.58 \pm 14.45$ & $7.12 \pm 1.03$ \\
F & & 7.13 & 6.65 & 6.11 & 5.29 \\
P & & 0.031 & 0.038 & 0.040 & 0.046 \\
\hline
\end{tabular}

\subsection{The Correlation Between Thyroid Function Index and Renal Function Index}

correlation with cystatin $\mathrm{C}$ and creatinine clearance, TSH, $\mathrm{TPOAb}, \mathrm{TGAb}$ is in positive correlation with 24-hour urinary microalbumin. (Table 3)

The correlation analysis shows that FT3, FT4 are in negative correlation with serum creatinine and in positive

Table 3. The Correlation between Thyroid Function Index and Renal Function Index.

\begin{tabular}{lllll}
\hline \multirow{2}{*}{ ThyroidFunction Index } & $\mathbf{r}$ & & & \\
\cline { 2 - 5 } & Cys-C & Serum creatinine & Ccr & 24-hour urinary microalbumin \\
\hline TSH & -0.201 & 0.198 & -0.176 & $0.325^{*}$ \\
FT3 & $0.553^{*}$ & $-0.516^{*}$ & $0.478^{*}$ & 0.164 \\
FT4 & $0.478^{*}$ & $-0.415^{*}$ & $0.406^{*}$ & 0.147 \\
TPOAb & 0.231 & 0.179 & 0.245 & $0.593^{*}$ \\
TGAb & 0.176 & 0.165 & 0.213 & $0.540^{*}$ \\
\hline
\end{tabular}

Note: $* \mathrm{P}<0.05$

\section{Discussions}

For a long time, the secondary renal disease caused by thyroid disease has not been paid enough attention in the clinic, which often leads to missed diagnosis and irreversible changes in renal function. Early detection and treatment of renal disease are beneficial to improve the prognosis of patients. According to previous studies, the incidence of AITD related nephropathy is $11 \% \sim 40 \%$, while in this paper, the incidence of HT patients complicated with renal injury is $13.46 \%$. TSH, $\mathrm{TPOAb}, \mathrm{TGAb}$ in renal injury group were distinctly higher than those in non-renal injury group, while FT3 and FT4 were distinctly lower than those in non-renal injury group, suggesting that the occurrence of renal injury in HT patients is closely related to thyroid function indexes.

Serum creatinine is an index of glomerular filtration function; however, in the early stage of renal disease, serum creatinine is usually in the normal range. Ccr is a better index of glomerular filtration function, which can determine glomerular injury earlier [8]. By comparing the renal function indexes of HT patients with different thyroid function states, it was found that from Hashimoto hyperthyroidism group to Hashimoto sub-hypothyroidism group, and then to Hashimoto hypothyroidism group, serum creatinine presented a gradually 
increasing trend, and Ccr presented a gradually decreasing trend. The reason may be that the strong activity of nitric oxide synthase in the renal of HT patients with hyperthyroidism increased the vasodilation effect of nitric oxide [9] and decreased the vasoconstriction effect [10], and then the patient was in a state of high hemodynamics, the renal filtration rate increased, creatinine excreted from urine increased, Ccr increased, and serum creatinine decreased; while the patients in Hashimoto hypothyroidism group had hypothyroidism, cardiac output decreased, blood flow was low, the glomerular filtration rate decreased, creatinine excreted with urine decreased, Ccr decreased and serum creatinine increased. Correlation analysis showed that FT3 and FT4 were negatively correlated with serum creatinine, and positively correlated with Ccr. With the decrease of FT3 and FT4 levels, glomerular filtration function was weakened, and the renal was in a low filtration state for a long time, which may lead to renal injury [11].

Cys-C is an index of renal dysfunction found in recent years, and its level is not affected by diet, height, age, inflammation, or other factors. The kidney is the only organ to remove Cys-C, which can be filtered freely through the glomerulus. Almost all $\mathrm{Cys}-\mathrm{C}$ in the original urine is reabsorbed and decomposed by the proximal renal tubules, and no longer enters the blood circulation again. It is a better index of glomerular filtration function than creatinine [12]. When the glomerular filtration rate decreases, the serum Cys-C level will increase significantly. This research shows that Cys-C in HT patients with hyperthyroidism is significantly increased, while Cys-C in HT patients with hypothyroidism is significantly decreased. Cys-C is positively correlated with FT3 and FT4, and its change trend is opposite to serum creatinine, which may be related to the increase of Cys-C secretion due to thyroid hormone [13]. The level of Cys-C in Hashimoto patients with hyperthyroidism is increased due to the increase of thyroid hormone; while in Hashimoto sub-hypothyroidism and hypothyroidism patients, thyroid hormone level is insufficient, the body is in a low blood flow state, glomerular filtration function is reduced, and the production and clearance of Cys-C are reduced. The decrease of Cys-C in serum may be due to the decrease of Cys- $\mathrm{C}$ production caused by the decrease of thyroid hormone, which is stronger than the increase of Cys-C caused by the decrease of the glomerular filtration function. Therefore, it is necessary to consider thyroid hormone's effect on the formation of Cys-C when using Cys-C to determine HT patients with renal injury.

Urinary microalbumin is one of the sensitive indexes of early glomerular injury, 24-hour urinary protein can accurately reflect the protein loss within one day, and is the golden standard for the detection of proteinuria [14]; This study found that 24-hour urinary microalbumin in Hashimoto hypothyroidism group and Hashimoto sub-hypothyroidism group was distinctly higher than that in other groups, and the correlation analysis suggested that TSH, TPOAb, and TGAb were positively correlated with 24-hour urinary microalbumin.
Previous studies have found that the thyrotropin receptor is expressed in a variety of extrathyroid tissues, including the kidney [15]. Both Hashimoto hypothyroidism group and Hashimoto sub-hypothyroidism group increased TSH, which could affect the renal by binding with its corresponding receptor [16]. TSH could also cause renal function injury by causing dysfunction of renal vascular endothelial cells [17], which may be the cause of increased urinary micro-protein. Besides, TPOAb and TGAb were found in the serum of HT patients, which combined with the corresponding antigen to form a circulating immune complex and deposit in the glomerulus. Or TPO and TG deposited in the glomerulus and combine with the corresponding antibody to form in situ complex [18]. The immune complex can activate complement through Classical pathway, and then form a membrane attack complex to destroy glomerular tissue. $\mathrm{C} 3 \mathrm{~b}$ produced in the process of complement activation can also be deposited in the glomerulus, causing renal function injury. Moreover, the cleavage fragments formed in the process of complement activation, such as $\mathrm{C} 3 \mathrm{a}$ and $\mathrm{C} 5 \mathrm{a}$, can result in a variety of inflammatory effects and further aggravate the occurrence of renal injury. The levels of TPOAb and TGAb in Hashimoto hypothyroidism groupand Hashimoto sub-hypothyroidism group are distinctly higher than those in Hashimoto hyperthyroidism group and Hashimoto normal group [19], which may be another reason for the increase of urinary protein in Hashimoto hypothyroidism group and Hashimoto sub-hypothyroidism group.

\section{Conclusions}

To sum up, the occurrence of renal injury in Hashimoto's thyroiditis is closely related to thyroid function, FT3 and FT4 affect renal function by affecting glomerular filtrationrate, with the decrease of FT3 and FT4 levels, glomerular filtration function was weakened, and the renal was in a low filtration state for a long time, which may lead to renal injury; TSH, $\mathrm{TPOAb}$ and TGAb lead to renal injury mainly by destroying glomerular filtration barrier, with the increase of TSH, $\mathrm{TPOAb}$ and TGAb levels, the urine microalbumin increased at 24 hours. So in the development process of the disease, HT patients should pay attention to the monitoring of renal function indexes, especially the patients with hypothyroidism and high antibody levels, to help the early detection and treatment of the renal injury.

\section{Acknowledgements}

This study was supported by a project grant from: science and technology research and development guidance plan project in cangzhou (Grant No. 172302039).

\section{References}

[1] Liao eryuan, YUAN Lingqing. Endocrinology and metabolism [M]. 4th Edition. Beijing: People's Medical Publishing House, 2019: 428. 
[2] Zhao Lian. The level of inflammatory marker in the serum of patients with Hashimoto thyroiditis [D]. [Master's thesis]. Suzhou: Suzhou University, 2017: 1.

[3] Amato AA, Santos GM, NevesFde A. Thyroid hormone action in chronic kidney disease [J]. CurrOpin Endocrinol Diabetes Obes, 2008, 15 (5): 459-465.

[4] Wei Xiaoqing. Clinical Analysis on Hypothyroidism and Renal Damage [D]. [Master's thesis]. Wuhu: Wan Nan Medical College, 2016: 1 .

[5] Gabalec F, Srbova L, Nova M, et al. Impact of Hashimoto's thyroiditis, TSH levels, and anti-thyroid antibody positivity on differentiated thyroid carcinoma incidence [J]. Endokrynol Pol, 2016, 67 (1): 48-53.

[6] GeJunbo, XuYongjian. Internal Medicine [M]. 8th Edition. Beijing: People's Medical Publishing House, 2013: 698.

[7] Dai Rongqin, Liu Yuzhi, Wang Fengling, et al. The incidence of hashimoto's thyroiditis in medical examination population in Huanghua City, Hebei Province [J]. Chinese Journal of Endemiology, 2019, 38 (2): 140-143.

[8] Wang Jianzhong, Zhang Man. Laboratory Diagnostics [M]. 8th Edition. Beijing: Peking University Medical Press, 2019: 356.

[9] Quesada A, Sainz J, Wangensteen R, et al. Nitric oxide synthase activity in hyperthyroid and hypo-thyroid rats [J]. Eur J Endocrinol, 2002, 147 (1): 117-122.

[10] Gunqor O, Kircelli F, Voroneanu L, et al. Hormones and arterial stiffness in patients with chronic kidney disease [J]. J Atheroscler Thromb, 2013, 20 (9): 698-707.

[11] Bulur O, Dal K, Ertugrul DT, et al. Renal function improves with the treatment of hypothyroidism [J]. Endocr Res, 2017, 42 (3): 246-251.

[12] Zhang Ke, Tong Shutao, Zhang Detai. Correlation between serum thyroid hormones and $\mathrm{Cr}$ and $\mathrm{Cys} \mathrm{C}$ in patients with initial thyroid dysfunction [J]. Laboratory Medicine, 2020, 35 (7): 691-694.

[13] Schmid C, Ghirlanda-Keller C, Zwimpfer C, et al. Triiodothyronine stimulates cystatin C production in bone cells [J]. Biochem Biophys Res Commun, 2012, 419 (2): 425-430.

[14] Wei Xiaoqing, Wang Hong, Qian Guangrong, et al. Correlation between serum thyroid patients with type 2 hormone levels and renal damage in diabetic nephropathy [J]. Medical Journal of West China, 2019, 31 (5): 774-777, 781.

[15] Briet C, Suteau-Courant V, Munier M, et al. Thyrotropin receptor, still much to be learned from the patients [J]. Best Pract Res Clin Endocrinol Metab, 2018, 32 (2): 155-164.

[16] Yang Sijue, Kang Jian, Guan Haixia. Research progress in the influence of thyroid dysfunction on chronic kidney disease [J]. Chinese Journal of Internal Medicine, 2021, 60 (1): 80-83.

[17] Das G, Taylor PN, Abusahmin H, et al. Relationship between serum thyrotropin and urine albumin excretion in euthyroid subjects with diabetes [J]. Ann Clin Biochem, 2019, 56 (1): 155-162.

[18] Li Di, Li Yan, PengRui. Study of renal damage associated with Graves disease [J]. Laboratory Medicine and Clinic, 2017, 14 (10): 1397-1398, 1401.

[19] Rongqin Dai, Yuzhi Liu, Jinbiao Zhang. The Research on the Correlation of Thyroid Function and Serum Immunological Indexes in Patients with Hashimoto's Thyroiditis [J]. American Journal of Life Sciences, 2020, 8 (2): 19-22. 